\title{
Erratum to: Anémie et cancer
}

Erratum to: Oncologie DOI:10.1007/s10269-011-2034-8

Référentiels interrégionaux en soins oncologiques de support - Anémie et cancer, page 332, il faut lire :

\section{Contributeurs}

Coordination

Didier Mayeur (ONCORIF)

Membres du groupe de travail
A.L. Couderc
N. Jovenin
A. Lerol
S. Rigaudeau
M. Vittot Manolis
D. Jaulmes

Relecture

F. Farsi (RRC-RA)

Contributeurs (participants aux ateliers des J2R du 02 décembre 2010)

Leila Ben Abdesselem (RRC-RA), Isabelle Bereder (ONCOPACA), Pierre Biron (RRC-RA), Sabine Brouers (Belgique), Michel Brun (ONCOPACA), Anne Laure Couderc (ONCOPACA), Yves de Muizon (RRC-RA), Gerard Dinne, Isabelle Duclot (RRC-RA), Marjorie Dumont (RRC-RA), Herve Gautier (ONCORIF), Veronique Guerin Meyer (ONCO PAYS DE LOIRE), Louis Guesnier (Belgique), Tawfiq Henni (ONCORIF), Nicolas Jovenin (ONCOCHA), Didier Mayeur (ONCORIF), Patrick Merlin (ONCOLOR), Henri Pierson (ONCOLOR), Florian Scotte (ONCORIF), Martine Vittot (ONCOPACA) 